\title{
Asymmetric Synthesis of Tetrahydrofurans Bearing Multiple Stereocenters
}

\section{Category}

Metal-Catalyzed

Asymmetric

Synthesis and

Stereoselective

Reactions

Key words

tetrahydrofurans

cycloaddition

palladium

SYNFACTuth<smiles>C=C[C@H]1COC(=O)O1</smiles>

$\mathrm{R}^{1}=\mathrm{Ar}$, Hetar, Alk

$\mathrm{R}^{2}=\mathrm{Ar}$, Hetar, Alk (one example) $\mathrm{R}^{3}=\mathrm{CN}, \mathrm{CO}_{2} \mathrm{Et}, \mathrm{CO}(t-\mathrm{Bu}), \mathrm{SO}_{2} \mathrm{Ph}$
Pd(dba) $)_{3} \cdot \mathrm{CHCl}_{3}(1 \mathrm{~mol} \%)$
ligand $(4 \mathrm{~mol} \%)$

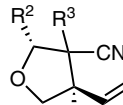

29 examples $68-99 \%$ yield up to $98 \%$ ee dr up to $19: 1$

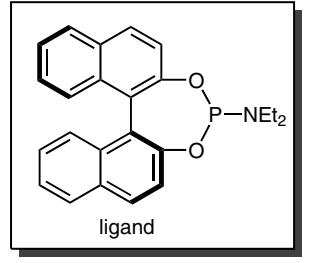

\section{Selected examples:}

Yield of combined diastereomers<smiles>C=C[C@]1(c2ccccc2)CO[C@@H](Pc2ccccc2)C1(C#N)C#N</smiles>
$98 \%$ yield $\mathrm{dr}=14: 1$<smiles>C=C[C@H]1COC(c2ccccc2)C1(C#N)C#N</smiles>
$90 \%$ yield $83 \%$ ee<smiles>C=C[C@]1(c2ccc(OC)cc2)CO[C@@H](c2ccccc2)C1(C#N)C#N</smiles>
95\% yield $93 \%$ ee
$\mathrm{dr}=13: 1$<smiles>[Mg]c1ccccc1</smiles><smiles>CCCCC1(O)CCCC1</smiles><smiles>C1=CC2COC2C2COCC12</smiles><smiles>c1ccccc1</smiles>
$97 \%$ yield $87 \%$ ee $\mathrm{dr}=6: 1$

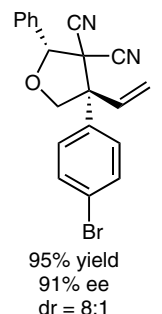

$\square$
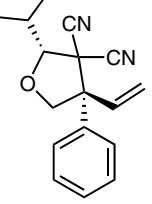

\section{$94 \%$ yield} $74 \%$ ee
$\mathrm{dr}=2: 1$
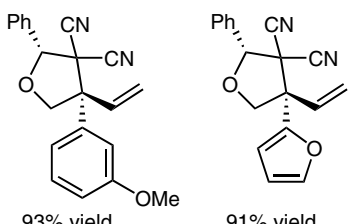

$93 \%$ yield
$92 \%$ ee $92 \%$ ee
$d r=6: 1$

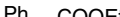<smiles>C=C[C@H]1COC(c2ccccc2)C1(OCC)OCC</smiles><smiles>c1ccccc1</smiles>

$98 \%$ yield $80 \%$ ee
$\mathrm{dr}=8: 1$

$$
\begin{gathered}
91 \% \text { yield } \\
92 \% \text { ee }
\end{gathered}
$$$$
\begin{aligned}
& 92 \% \text { ee } \\
& \mathrm{dr}=7: 1
\end{aligned}
$$

$\mathrm{Ph}$

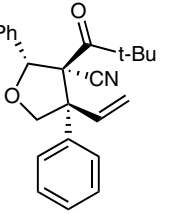

$93 \%$ yield $93 \%$ ee
$\mathrm{dr}=11: 1$

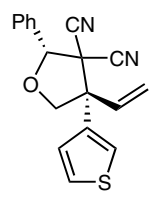

$95 \%$ yield $92 \%$ ee

Proposed mechanism:

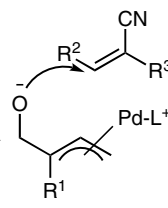<smiles>C=CCCCCC(=C)COC(PC)C(C#N)C#N</smiles><smiles>CC</smiles><smiles>[R]C1OC[C@@]([18F])(C=C)[C@@H]1C#N</smiles>

Significance: Transition-metal-catalyzed reactions that form multiple stereocenters in a single operation are particularly challenging with respect to absolute and relative stereocontrol. The authors report an efficient reaction between vinylethylene carbonates and doubly activated Michael acceptors, yielding tetrahydrofurans bearing two or three stereocenters, with good to high enantioselectivity.

sYNFACTS Contributors: Mark Lautens, Thomas Johnson Synfacts 2014, 10(11), $1161 \quad$ Published online: 20.10.2014 DOI: 10.1055/s-0034-1379274; Reg-No.: L12714SF
Comment: This reaction is an innovative variation on the theme of decarboxylative allylation (see Review below). The reaction shows a high functional group tolerance with respect to both reaction partners and proceeds under mild conditions. Although the total yields are consistently high, the diastereomeric ratios are variable. The reaction was scaled-up to $2.2 \mathrm{mmol}$ scale.

Review: J. A. Tunge and co-workers Chem. Rev. 2011, 111, 1846-1913. 